\title{
The Fluorescence Correction of Multilayer Materials for Quantitative X-ray Microanalysis
}

\author{
Yu Yuan ${ }^{1}$, Hendrix Demers ${ }^{1}$, and Raynald Gauvin ${ }^{1}$ \\ 1. Department of Mining and Materials Engineering, McGill University, Montreal, Quebec, Canada
}

In the procedure of quantitative $\mathrm{x}$-ray microanalysis, the effect of $\mathrm{x}$-ray fluorescence on the emitted $\mathrm{x}$ ray intensities is usually ignored because of its tiny magnitude, i.e., for large homogenous sample. However, its contribution can be significant in some cases like thin film on a substrate [1,2]. A program was developed for both characteristic and bremsstrahlung x-ray fluorescence correction based on MC xray, a Monte Carlo simulation software [3]. At present, our program can be applied to multilayer materials.

MC X-ray is a powerful software based on Monte Carlo method used to simulate the electron trajectories and electron-induced $\mathrm{x}$-ray emission. Using $\mathrm{MC} \mathrm{x}$-ray, the depth distribution of generated $\mathrm{x}$-ray intensities (both characteristic and bremsstrahlung) can be easily obtained, which is used as the input of our program. Take the calculation of secondary x-ray intensity of element $A$ as an example. For certain $\mathrm{X}$-ray B, when its energy is greater than the ionization energy of element A, secondary $\mathrm{x}$-ray intensity of element $\mathrm{A}$ at mass depth $\rho t_{A}$ emitted by $\mathrm{x}$-ray $\mathrm{B}$ in each slice are calculated and summed up to get $I_{f, A}\left(\rho t_{A}\right)$, which is the total generated secondary $\mathrm{X}$-ray intensity at mass depth $\rho t_{A}$. Following that, the $\mathrm{X}-$ ray intensity is integrated at all mass depths to acquire the total fluorescence X-ray intensity $I_{f, A}$. The program outputs the ratio of $I_{f, A}$, secondary x-ray intensity to $I_{p}$, the primary intensity excited by the electrons. Since only the secondary $x$-ray intensity from certain depth is calculated at a time the program can be applied to any kind of multilayer materials. Assuming that the emission of bremsstrahlung x-rays is isotropic, the x-ray intensity excited by the bremsstrahlung x-rays is calculated in the same way as characteristic $\mathrm{x}$-rays.

To test the accuracy of the program, the iron nickel alloy (Fe-Ni) bulk materials is studied. Figure1 a) compares computed $\phi(\rho z)$ curves of Fe Ka line with or without fluorescence. It is observed that the intensity of characteristic fluorescence is much greater than that of bremsstrahlung fluorescence, which is because of the strong absorption of Ni. Figure $1 \mathrm{~b}$ ) shows the variation of ratio of secondary x-ray intensity to primary intensity of Fe Ka line with different concentration at $30 \mathrm{kV}$ compared with DTSAII [4]. A small contribution from bremsstrahlung is observed, which only slightly changes with iron concentration while the characteristic fluorescence decreases rapidly when the concentration of iron increases. Besides, calculation results from our program are really close with DTSA results, while the slight difference can be attributed to different input data (including mass absorption coefficient and mass density) used.

As an example of applying for multilayer materials, an aluminum film on silicon substrate is considered. As shown in Figure 2 is the variation of intensity ratio $\mathrm{I}_{\mathrm{f}} / \mathrm{I}_{\mathrm{p}}$ of $\mathrm{Al} \mathrm{Ka}$ line and primary intensity ratio with film thickness at $15 \mathrm{kV}$. As the increasing of film thickness, the effect of fluorescence on the total x-ray intensity (mainly characteristic fluorescence) decreases, which is because of the decrease of Si X-ray intensity, as shown in the green curve in Figure 2.

In the future, the fluorescence correction is going to be extended to apply for heterogeneous material to 
deal with complicated cases like interface between weld metal and heat affected zone.

\section{References:}

[1] J. Armstrong, and P. Buseck, X-Ray Spectrometry, 14.4 (1985), p. 172-182.

[2] Y. Yuan et al, Microscopy and Microanalysis (2016) p. 400-401.

[3] R. Gauvin and P. Michaud, Microscopy and Microanalysis, 15 (2009) 488-489.

[4] Ritchie, N. W. Spectrum Simulation in DTSA-II, Microscopy and Microanalysis, 15 (2009) 454-468.
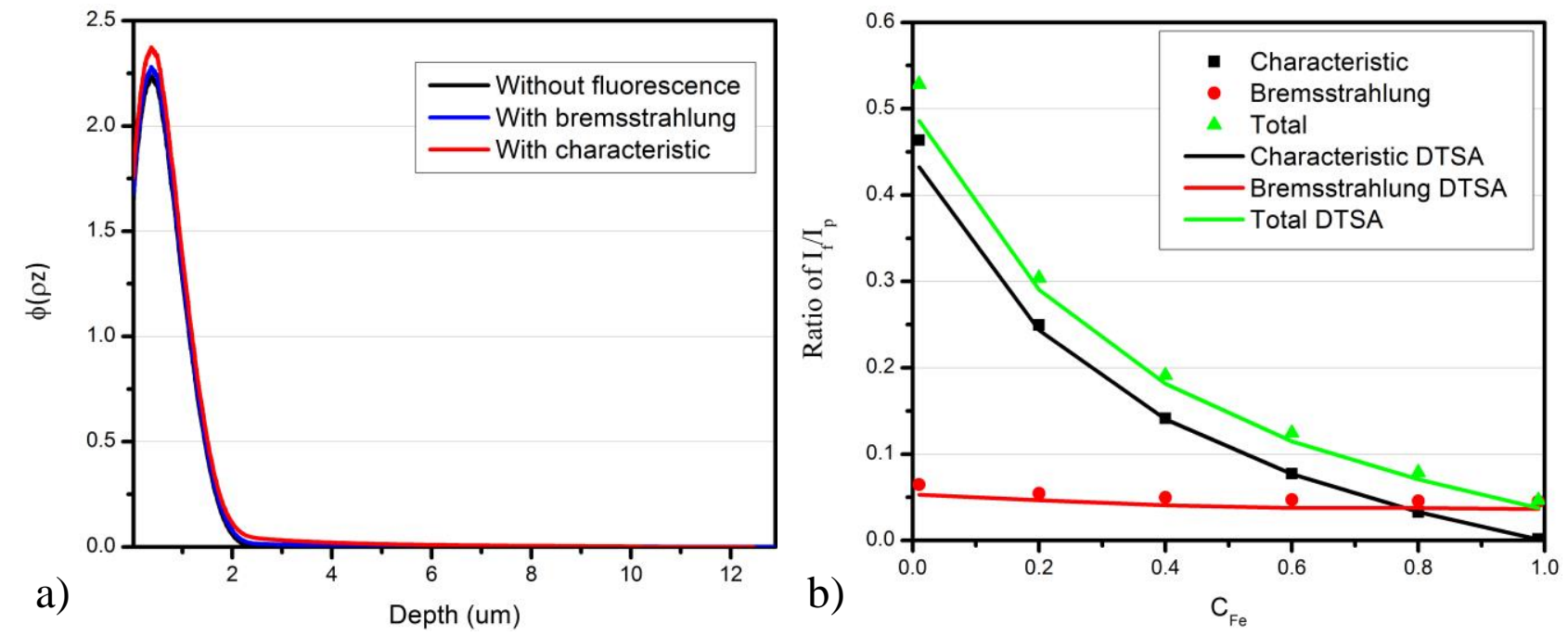

Figure 1. a) Depth distribution curve of $\mathrm{Fe} \mathrm{Ka}$ line in Fe-Ni alloy with concentration of $\mathrm{Fe} 40 \%$ at 30 $\mathrm{kV}$ for electron excited $\mathrm{x}$-ray, $\mathrm{x}$-ray fluorescence by characteristic $\mathrm{x}$-ray, and $\mathrm{x}$-ray fluorescence by bremsstrahlung x-rays. b) Variation of the ratio $\mathrm{I}_{\mathrm{f}} / \mathrm{I}_{\mathrm{p}}$ for $\mathrm{Fe} \mathrm{Ka}$ line in $\mathrm{Fe}-\mathrm{Ni}$ alloy with different concentration at $30 \mathrm{kV}$.

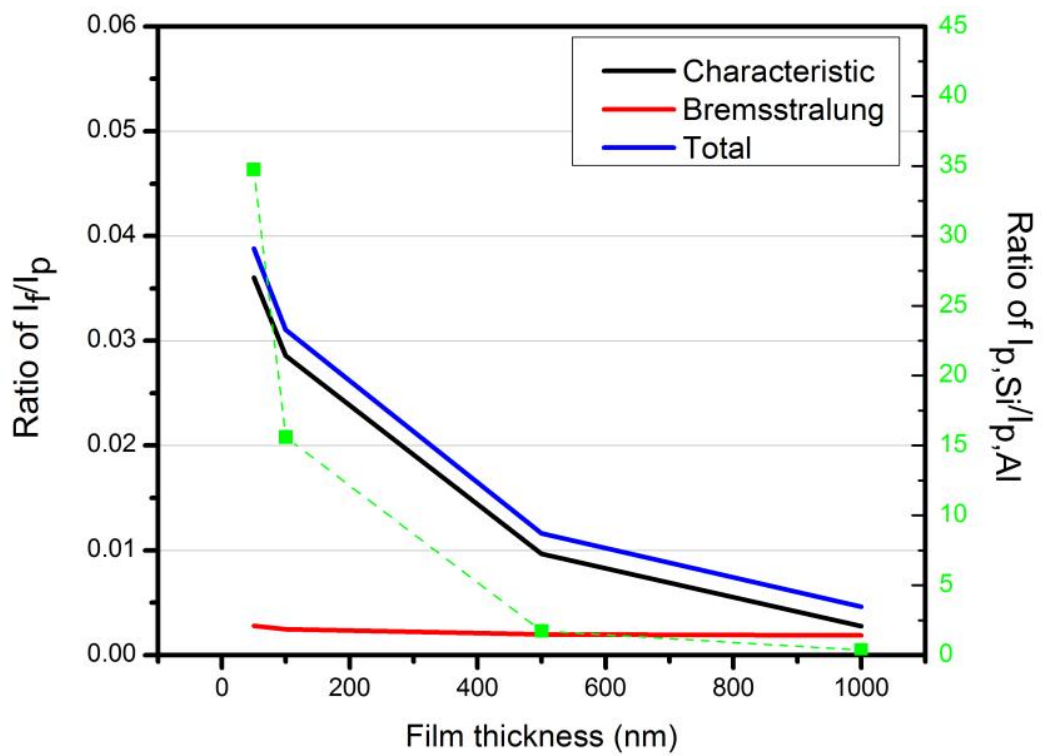

Figure 2. Variation of intensity ratio $I_{f} / I_{p}$ of $A l$ Ka line and primary intensity ratio of $I_{p, S i} / I_{p, A l}$ with film thickness in $\mathrm{Al}$ thin film on $\mathrm{Si}$ substrate at $15 \mathrm{kV}$. 